\title{
Anaesthetic management of a labouring parturient with Brugada Syndrome
}

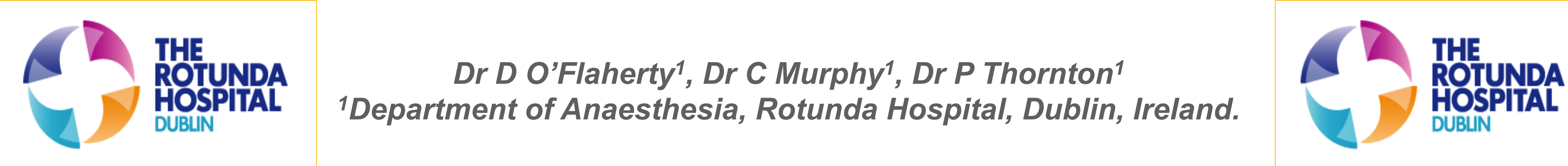

\section{Introduction}

- Brugada syndrome is an autosomal dominant ion channelopathy affecting mainly cardiac sodium ion channels, which may lead to ventricular tachyarrhythmias and ultimately sudden cardiac death [1].

- It is characterised by typical ECG changes which can be transient (see figure1)

\section{Modulating factors}

- The syndrome may be unmasked by drugs used perioperatively, fever, surgical insult and vagal stimulation [2]

- Local anesthetics with slow dissociation characteristics such as Bupivacaine, have been discouraged due to concerns regarding unmasking the Brugada phenotype and potential precipitation of ventriular tachyarrythmias.

- Evidence suggests that Lignocaine is safe when used in low doses $[3,4]$.

\section{Case Report}

A 19 year old primigravida with Type 1 Brugada Syndrome was referred in early pregancy for anaesthetic assessment at high risk clinic.

Following multidisplinary team review, consensus regarding anaesthesia management in labour was agreed (Remifentanil PCA, followed by combined spinal epidural (CSE) if required). Limited dose of bupivacaine with avoidance of background bupivacaine infusion was advised and intermittent epidural top-up of lignocaine would be given as needed.

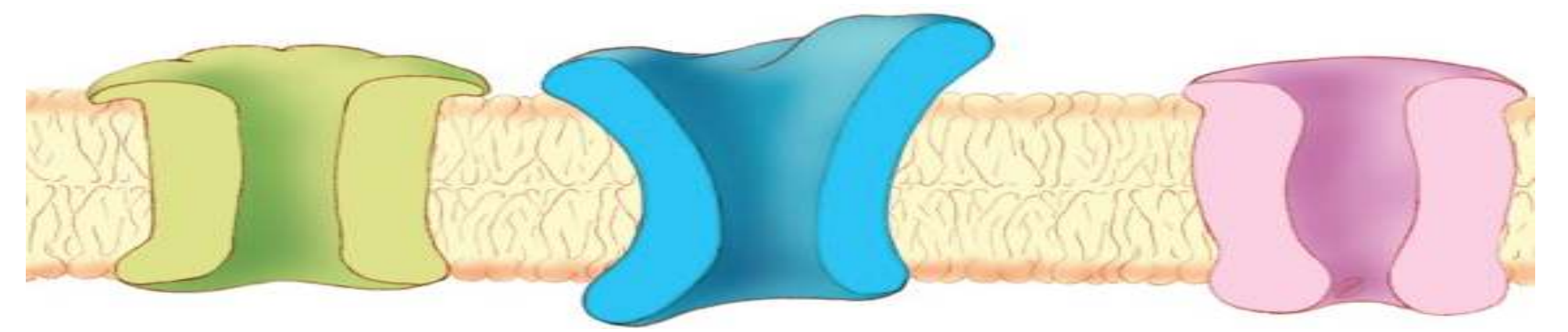

At 38 weeks gestation the patient presented with lower abdominal pains. Following induction of labour a Remifentanil PCA was commenced. When analgesia became unsatisfactory, a CSE was performed. Intrathecal bupivacaine $2.5 \mathrm{mg}$ with fentanyl $20 \mathrm{mcg}$ initially provided adequate analgesia with no untoward effect. Subsequent epidural top-ups of $5 \mathrm{mls}$ of $1 \%$ and $2 \%$ Lignocaine failed to achieve satisfactory analgesia. Following unsuccessful repeat CSE replacement, a repeat single intrathecal dose was administered for patient distress. The patient shortly delivered a healthy baby boy. No peripartum cardiac arrhythmias were recorded.

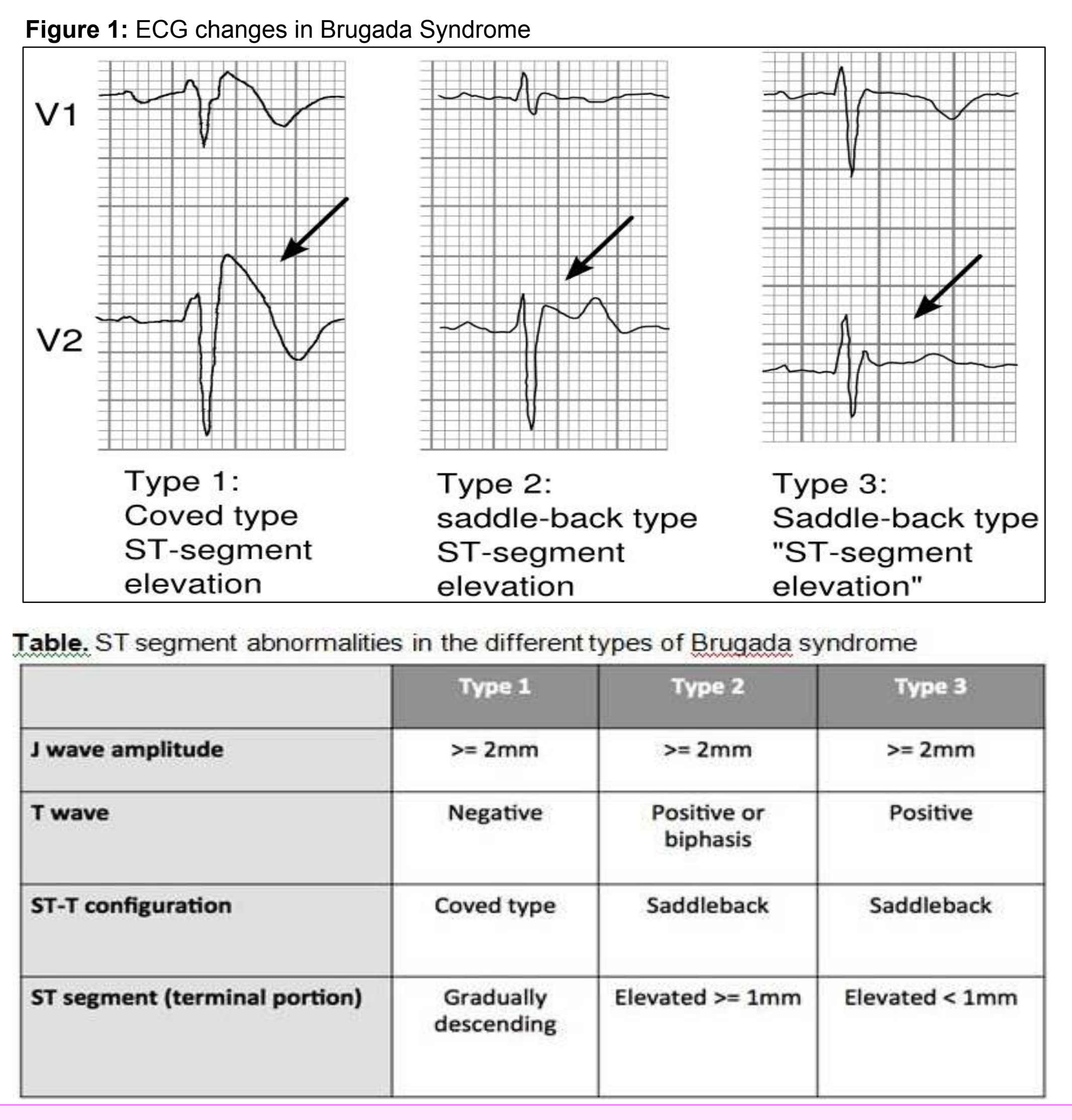

\section{Discussion}

- Brugada labouring parturients pose significant anaesthetic challenges.

- In our case, we were faced with conflicting priorities: minimize sodium channel blockade yet provide adequate analgesia for the duration of her labour.

- Evidence suggests that low dose bupivacaine is not a contraindication. Intrathecal bupivacaine for analgesia or caesarean delivery appears safe and should be considered.

- For this reason, a spinal catheter would seem a resonable option for a patient with Brugada syndrome for labour ward analgesia.

- Pyrexia and vagal stimulation should be actively avoided

1. Vohra Jitendra et al. Update on the diagnosis and management of Brugada Syndrome. Heart, Lung and Circulation, Volume 24 , Issue 12 , $1141-1148$

2. Sorajja D, Ramakrishna H, Poterack A K, Shen WK, Mookadam F. Brugada syndrome and its relevance in the perioperative period. Ann Card Anaesth 2015;18:403-13

3. Carey, S.M, Hocking, G. Brugada syndrome - a review of the implications for the

Anaesthetist. Anaesth Intensive Care 2011; 39 : 571-577

4. Postema PG, Wolpert C, Amin AS et al Drugs and Brugada syndrome patients: review of the literature, recommendations, and an upto-date website (www.brugadadrugs.org). Heart Rhythm 2009;6:1335-41 